\title{
Advances and understanding pitfalls of laparoscopic transhiatal esophagectomy with en bloc mediastinal lymph node dissection
}

\author{
Atsushi Shiozaki, Hitoshi Fujiwara, Hirotaka Konishi, Hiroki Shimizu, Michihiro Kudou, Tomohiro Arita, \\ Toshiyuki Kosuga, Ryo Morimura, Yoshiaki Kuriu, Hisashi Ikoma, Takeshi Kubota, Kazuma Okamoto, \\ Eigo Otsuji
}

Division of Digestive Surgery, Department of Surgery, Kyoto Prefectural University of Medicine, Kyoto 602-8566, Japan.

Correspondence to: Dr. Atsushi Shiozaki, Assistant Professor, Division of Digestive Surgery, Department of Surgery, Kyoto Prefectural University of Medicine, 465 Kajii-cho, Kamigyo-ku, Kyoto 602-8566, Japan. E-mail: shiozaki@koto.kpu-m.ac.jp

How to cite this article: Shiozaki A, Fujiwara H, Konishi H, Shimizu H, Kudou M, Arita T, Kosuga T, Morimura R, Kuriu Y, Ikoma $\mathrm{H}$, Kubota T, Okamoto K, Otsuji E. Advances and understanding pitfalls of laparoscopic transhiatal esophagectomy with en bloc mediastinal lymph node dissection. Mini-invasive Surg 2020;4:50. http://dx.doi.org/10.20517/2574-1225.2020.31

Received: 16 Mar 2020 First Decision: 10 Jun 2020 Revised: 1 Jul 2020 Accepted: 22 Jul 2020 Published: 15 Aug 2020

Academic Editor: Itasu Ninomiya Copy Editor: Cai-Hong Wang Production Editor: Jing Yu

\begin{abstract}
We began performing mediastinal lymph node dissection using the laparoscopic transhiatal approach in 2009. Following the initiation of the single-port mediastinoscopic cervical approach in 2014, we developed a technique for transmediastinal radical esophagectomy without a thoracic approach. We herein describe our surgical procedures for en bloc mediastinal lymph node dissection by the laparoscopic transhiatal approach with a focus on pitfalls. We opened the esophageal hiatus and the working space was secured using long retractors. During division of the right crus of the diaphragm, we made efforts to avoid damaging the left hepatic vein and inferior vena cava. Dissection of the posterior plane of the pericardium was extended to the cranial side, and the bilateral inferior pulmonary veins were identified. To avoid misorientation, the posterior plane was initially extended along the long axis of the esophagus. The anterior and posterior sides of the posterior mediastinal lymph nodes were then both dissected. These lymph nodes were lifted in a sheet-like form and then cut along the borderline of the left mediastinal pleura. The right side of the mediastinal lymph nodes was then dissected. To avoid damaging the arch of the azygos vein, it was identified at the dorsal side of the right main bronchus prior to lymph node dissection. This procedure decreased the total operative time, total operative bleeding, and postoperative respiratory complications without reducing the quality of lymphadenectomy. In conclusion, the procedure described herein resulted in a good surgical view and safe en bloc mediastinal lymph node dissection. A detailed understanding of mediastinal 3D anatomy and specific pitfalls is crucial for the successful use of this approach.
\end{abstract}

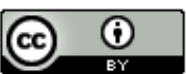

(C) The Author(s) 2020. Open Access This article is licensed under a Creative Commons Attribution 4.0 International License (https://creativecommons.org/licenses/by/4.0/), which permits unrestricted use, sharing, adaptation, distribution and reproduction in any medium or format, for any purpose, even commercially, as long as you give appropriate credit to the original author(s) and the source, provide a link to the Creative Commons license, and indicate if changes were made.

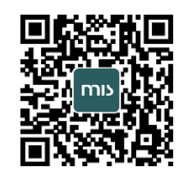


Keywords: Transmediastinal radical esophagectomy, laparoscopic transhiatal approach, pitfall

\section{INTRODUCTION}

Transthoracic esophagectomy with mediastinal lymphadenectomy has been the standard procedure for esophageal squamous cell carcinoma ${ }^{[1,2]}$. However, esophagectomy via right thoracotomy is highly invasive, and respiratory morbidity is still one of the most common complications ${ }^{[3]}$. Since Orringer and Sloan ${ }^{[4]}$ reported the clinical application of transhiatal esophagectomy, it has been broadly performed because it prevents respiratory complications. Although laparoscopic transhiatal esophagectomy, initially described by DePaula et al. ${ }^{[5]}$, has also been performed ${ }^{[5-7]}$, a technique for mediastinal lymphadenectomy had not yet been established because of the limited surgical view and difficulties associated with surgical procedures.

We began performing esophagectomy using the laparoscopic transhiatal approach for esophageal cancer in 2009 to reduce the duration of one-lung ventilation, and, to date, more than 400 patients have undergone our method during various esophageal surgical procedures ${ }^{[8-11]}$. We noted the advantages of this approach, and developed a novel technique for lower mediastinal lymph node dissection ${ }^{[8,10]}$. By applying the same concept to middle mediastinum, we developed a new procedure for subcarinal lymph node dissection using the laparoscopic transhiatal approach ${ }^{[12,13]}$. We also started using the single-port mediastinoscopic cervical approach in 2014, and developed a simple technique for transmediastinal radical esophagectomy without a thoracic approach (more than 200 patients) ${ }^{[14-17]}$.

We herein describe our surgical procedures for en bloc resection of the middle and lower mediastinal lymph nodes by the laparoscopic transhiatal approach, with a focus on pitfalls for safe surgery.

\section{SURGICAL PROCEDURES AND PITFALLS}

\section{Position, port placement, and devices}

Patients were placed in the supine position, and we initially performed cervical and upper mediastinal lymphadenectomy using the left cervical single port technique ${ }^{[14-16]}$. We recently performed middle mediastinal lymph node dissection via the cervical approach. Abdominal surgery was conducted using hand-assisted laparoscopic surgery (HALS), followed by lower mediastinal surgeries using the laparoscopic transhiatal approach. In cases in which middle mediastinal lymph node dissection was difficult to perform via the cervical approach, the laparoscopic transhiatal approach was employed.

We made an incision $(70 \mathrm{~mm}$ ) in the upper abdomen and inserted a lap disc (regular) (Ethicon, Cincinnati, $\mathrm{OH}, \mathrm{USA})^{[11]}$. We introduced three $12-\mathrm{mm}$ ports (right side of the umbilicus, left hypochondrium, and left flank), and one 5-mm port (left side of the umbilicus) for a flexible laparoscope ${ }^{[11]}$. The surgeon stayed at the patient's right side, and the 12 - $\mathrm{mm}$ port in the right side of the umbilicus was chiefly used for surgery. The assistant stayed on the left side, and two long retractors were inserted and used from ports in the left abdomen (UMIHIRA Co., Ltd., Japan). The scopist remained at the patient's groin [Figure 1] ${ }^{[11]}$.

\section{Approach to the esophageal hiatus}

Carbon dioxide was introduced into the abdominal space, and pneumoperitoneal pressure was held at $10 \mathrm{mmHg}^{[8-12]}$. We opened the esophageal hiatus, and carbon dioxide was introduced into the mediastinum. The use of long sealing devices is important for the laparoscopic transhiatal approach. The surgical view in the mediastinum was maintained by the surgeon's left hand, two long retractors, and pneumomediastinal pressure ${ }^{[11]}$. The bilateral mediastinal pleura were preserved as much as possible because pneumomediastinal pressure is essential for securing a narrow mediastinal surgical space. 


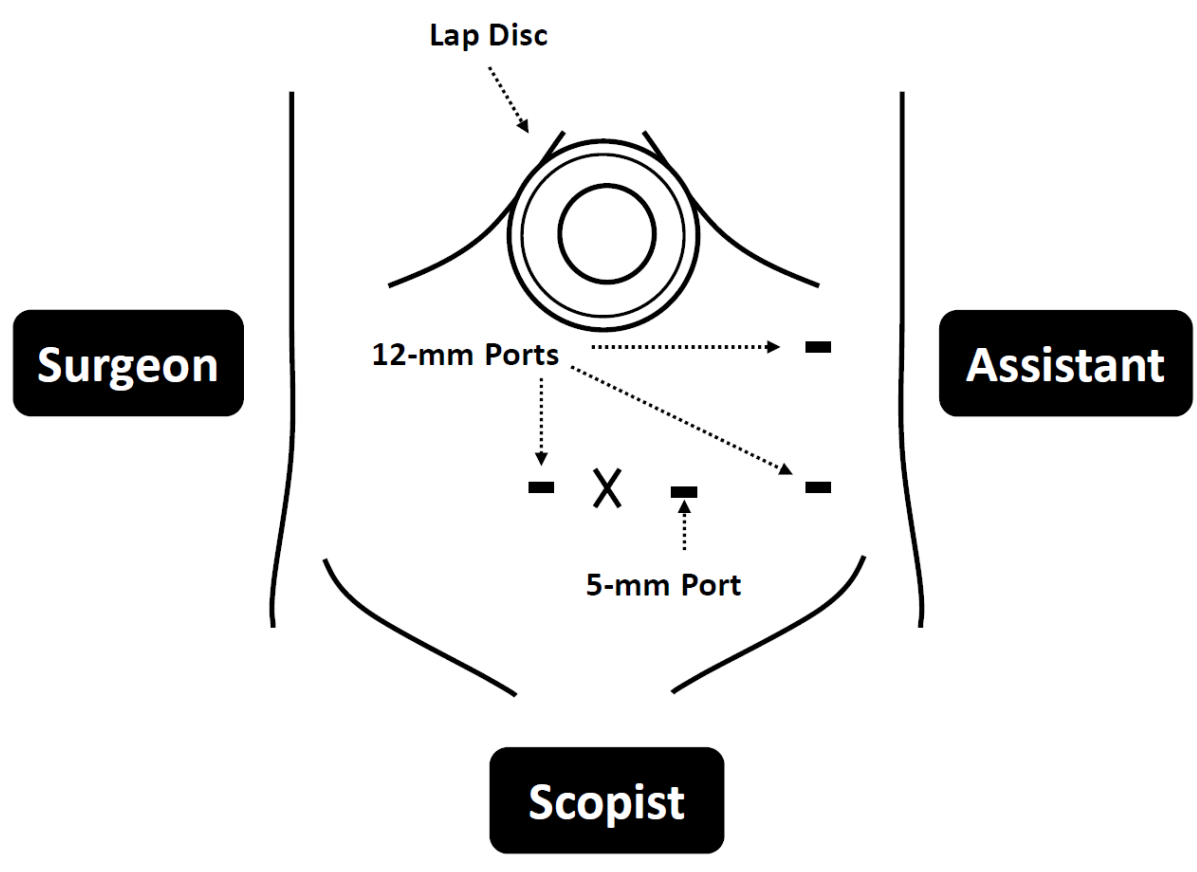

Figure 1. Intraoperative view of ports and incision locations on the abdomen ${ }^{[11]}$. A lap disc was inserted into the upper abdomen. Three 12-mm ports were introduced (right side of the umbilicus, left hypochondrium, and left flank), and one 5-mm port for a flexible laparoscope was inserted into the left side of the umbilicus

\section{Pitfall}

Operability by the HALS technique was very good at the left side of the esophageal hiatus, but was poor at the right side. Therefore, to obtain a sufficient surgical view of the right inferior mediastinal space, we divided the right crus of the diaphragm [Figure 2A]. Following its division, the esophagus was moved to the dorsal side. In this step, we made every effort to avoid damaging major vessels, such as the left hepatic vein and inferior vena cava, which are located near the right crus of the diaphragm [Figure $2 \mathrm{~B}$ ]. A detailed understanding of 3D images of these major vessels preoperatively is important.

\section{Exposure of the pericardium and inferior pulmonary vein}

In the inferior mediastinal space, we divided pericardial adipose tissue and exposed the pericardium. Dissection of the posterior plane of the pericardium was extended to the cranial side, and the bilateral inferior pulmonary veins were identified. This plane was extended to the left side of the esophagus, and abruption of the anterior sides of the posterior mediastinal lymph nodes was conducted ${ }^{[8-12]}$.

\section{Pitfall}

In this step, a detailed understanding of $3 \mathrm{D}$ images of the left inferior pulmonary vein is crucial. The pericardium was initially exposed, and this surgical plane was extended. Since the extension of this surgical plane to the left side in advance may separate the ventral side of the inferior pulmonary vein [Figure $3 \mathrm{~A}$ and $\mathrm{B}$ ], it was important to initially extend the plane along the long axis of the esophagus [Figure $3 \mathrm{C}$ and $\mathrm{D}$ ]. By extending the plane bilateral side, the dorsal side of the inferior pulmonary vein was clearly identified [Figure $3 \mathrm{C}$ and $\mathrm{D}]$.

\section{Subcarinal and main bronchus lymph node dissection}

In cases that underwent subcarinal lymph node dissection using the laparoscopic transhiatal approach, dissection of the posterior plane of the pericardium was extended to the level of the carina using a long sealing device, and the anterior side of the subcarinal lymph nodes and those of the bilateral main bronchi were dissected. 

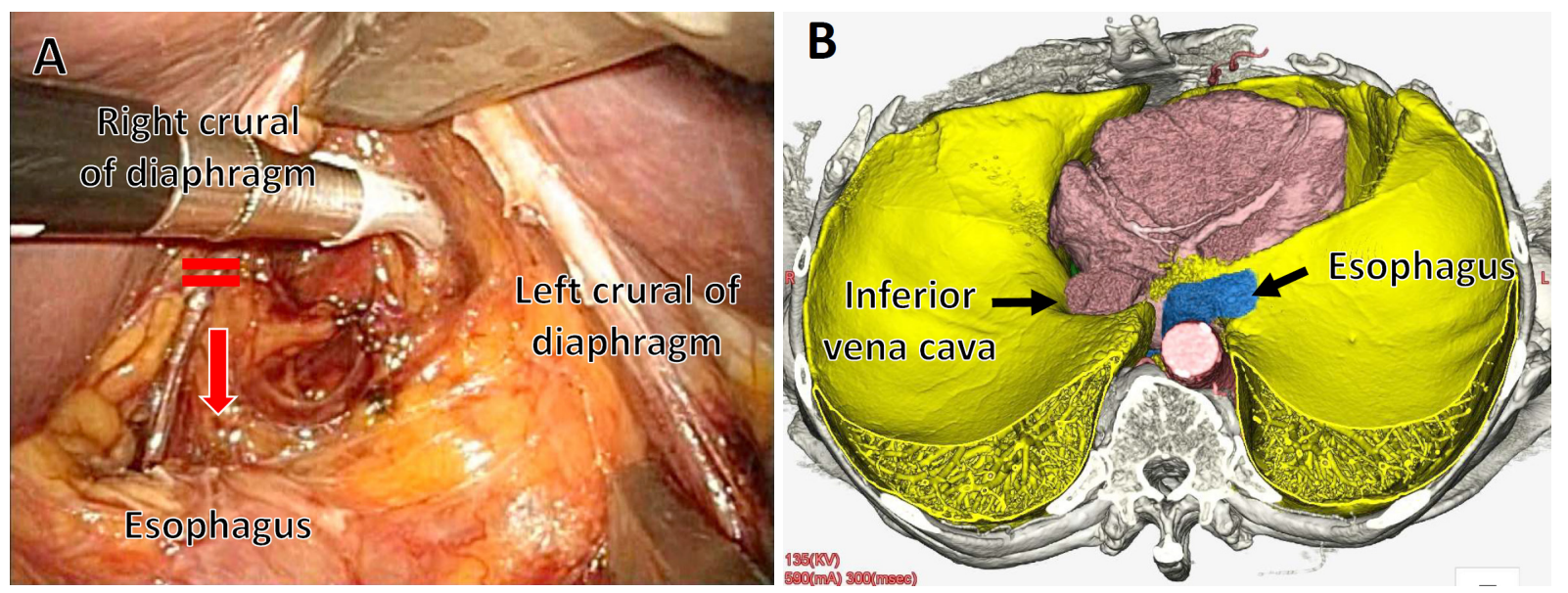

Figure 2. Pitfall around the right crus of the diaphragm. After cutting the right crus of the diaphragm (red double line), the esophagus was moved to the dorsal side (red arrow), and a sufficient surgical view of the inferior mediastinal space was obtained (A); at this point, major vessels, such as the inferior vena cava, were located near to the right crus of the diaphragm (B)
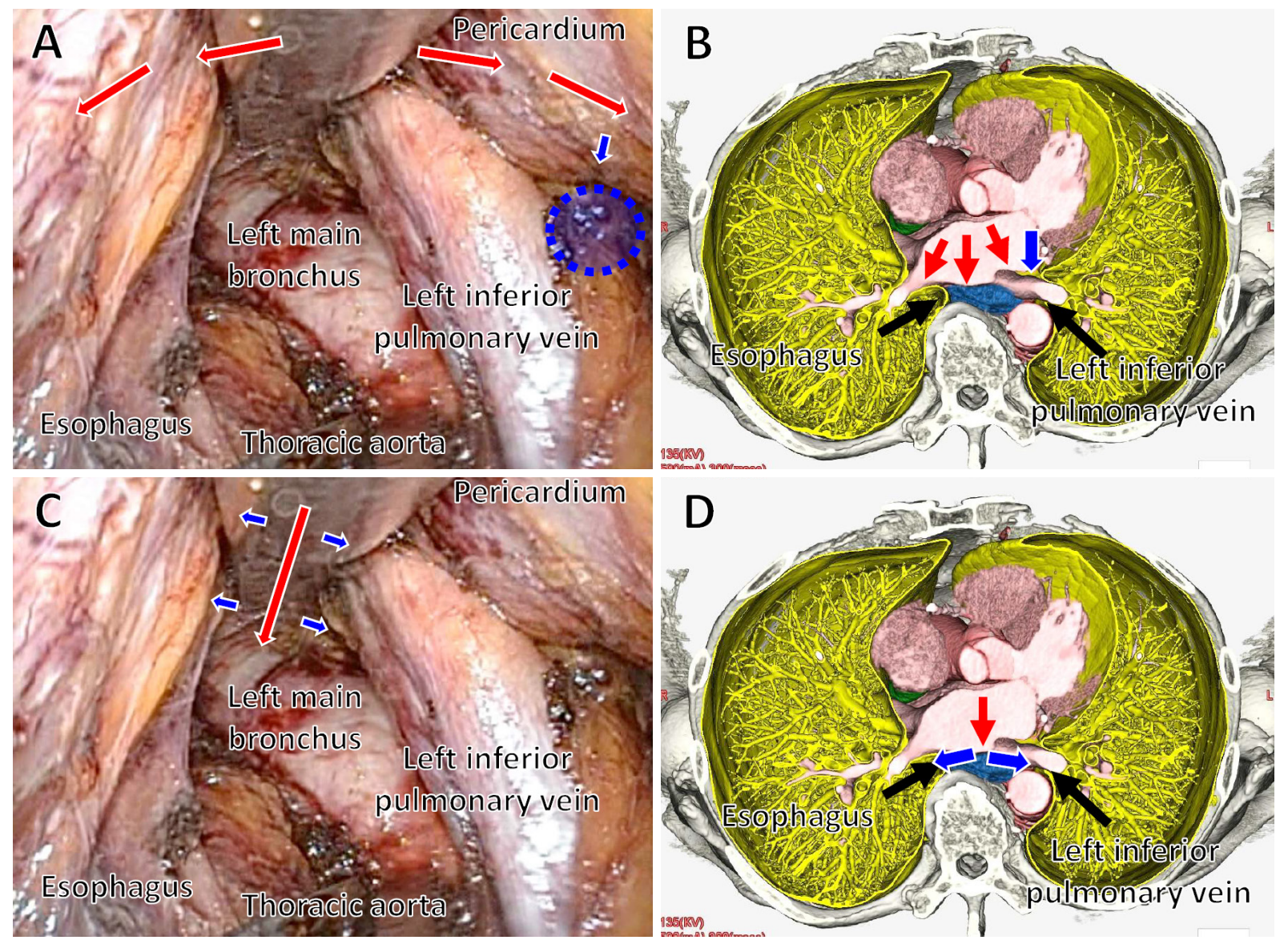

Figure 3. Pitfall around the left inferior pulmonary vein. The pericardium was initially exposed, and the surgical plane was extended. The extension of this plane to the bilateral side in advance (red arrows) may separate the ventral side of the left inferior pulmonary vein (blue arrows and circle) (A, B); to avoid misorientation, it was important to initially extend the plane along the long axis of esophagus (red arrows) (C, D). By extending the plane to the bilateral side (blue arrows), the dorsal side of the inferior pulmonary vein was certainly identified 


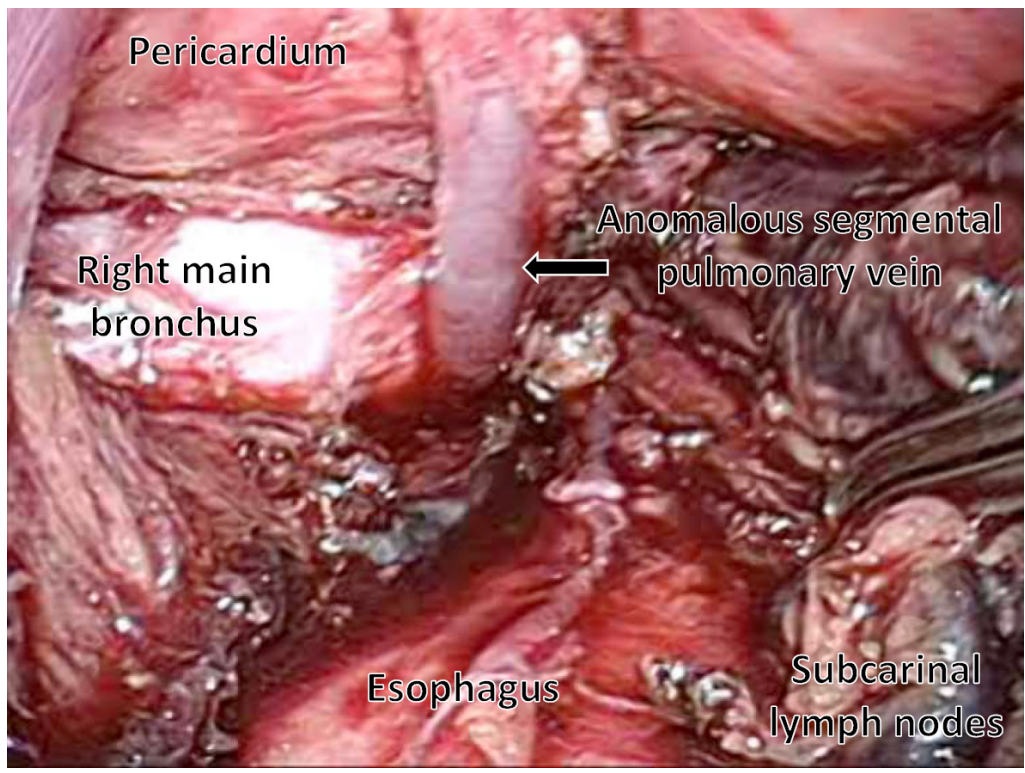

Figure 4. Anomalous pulmonary vein. An aberrant segmental vein in the right upper lobe that independently drained into the left atrium was identified (arrow). This anomalous vein penetrated the subcarinal lymph nodes and crossed behind the right main bronchus

\section{Pitfall}

In subcarinal dissection using the laparoscopic transhiatal approach, we need to consider anomalies of the pulmonary vein ${ }^{[18]}$. We encountered and reported the rare abnormality of an aberrant segmental vein in the right upper lobe that independently drained into the left atrium ${ }^{[18]}$. This anomalous vein penetrated the subcarinal lymph nodes and crossed behind the right main bronchus [Figure 4]. Although the anomalous pulmonary vein in the present case was not diagnosed preoperatively, our surgical procedure enabled the intraoperative identification of this vein and safe en bloc subcarinal dissection ${ }^{[18]}$.

\section{Abruption of the ventral side of the thoracic aorta}

We exposed the adventitia of the thoracic aorta at the level of the crural diaphragm, and dissected the anterior side of the thoracic aorta to the cranial side. The roots of the proper esophageal arteries were identified [Figure 5A] and divided using the long sealing device ${ }^{[8-12]}$.

\section{Pitfall}

When the proper esophageal arteries were divided using the sealing device, the assistant needed to decrease tension by the long retractor in order to avoid arterial damage [Figure 5B].

\section{Dissection of the left side of posterior mediastinal lymph nodes}

After these procedures, the anterior and posterior sides of the posterior mediastinal lymph nodes, including the thoracic para-aortic and left pulmonary ligament lymph nodes, were both dissected. These lymph nodes were lifted in a sheet-like form and cut along the borderline of the left mediastinal pleura, and, thus, the posterior mediastinal lymph nodes were dissected en $b l o c^{[8-12]}$ [Figure 6]. In cases that underwent middle mediastinal lymph node dissection using the laparoscopic transhiatal approach, this incision was extended to the left pulmonary hilum and the lymph nodes were dissected from the left main bronchus.

\section{Dissection of the right side of mediastinal lymph nodes}

In the dissection of the right side, an incision was made while lifting the right mediastinal pleura in a sheet-like form. In cases that underwent middle mediastinal lymph node dissection using the laparoscopic transhiatal approach, the incision was extended to the right pulmonary hilum, and the lymph nodes were 

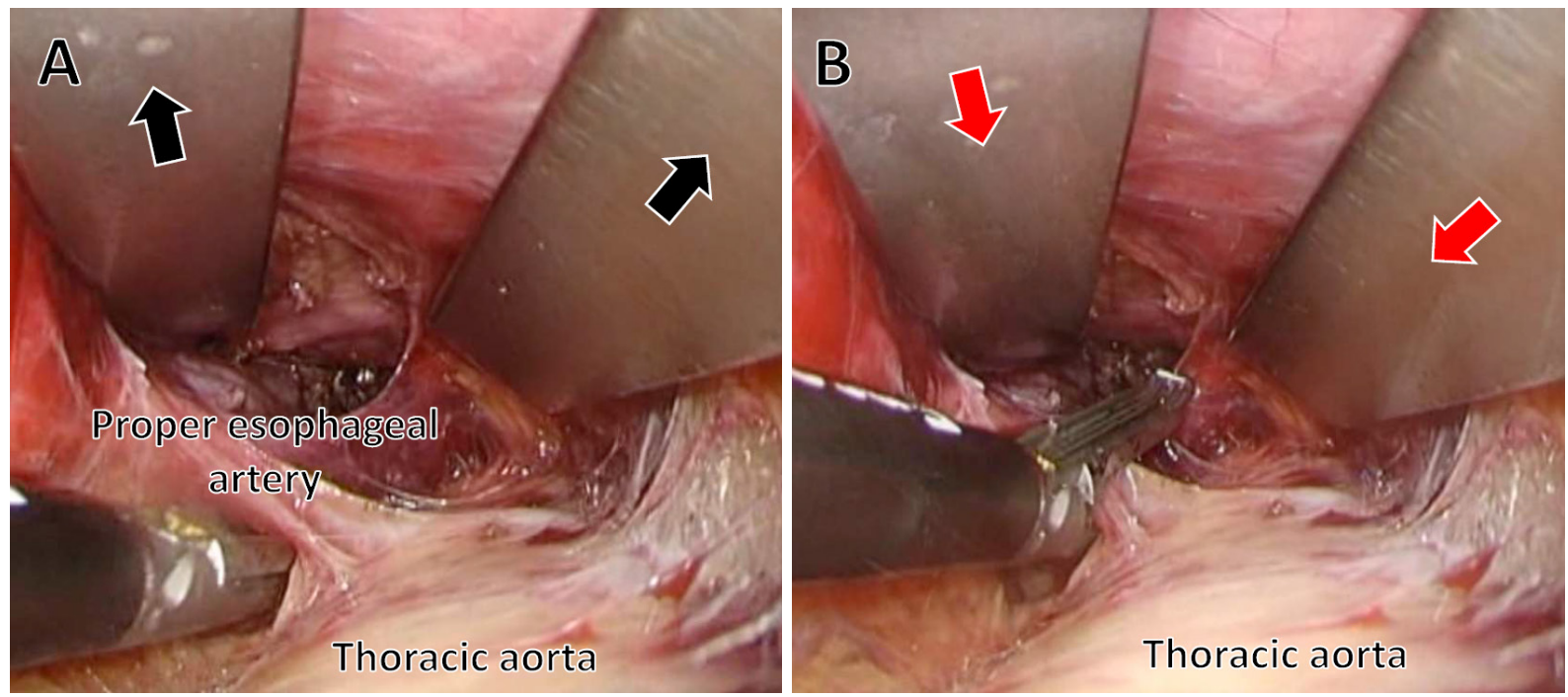

Figure 5. Pitfall around proper esophageal arteries. The adventitia of the thoracic aorta was exposed, and dissection of the anterior side of the thoracic aorta to the cranial side was performed. The roots of the proper esophageal arteries were identified. Black arrows showed the direction of tension given by the long retractor ( $A$ ); when the proper esophageal arteries were divided using the sealing device, the assistant needed to decrease tension by the long retractor (red arrows) to avoid arterial damage (B)

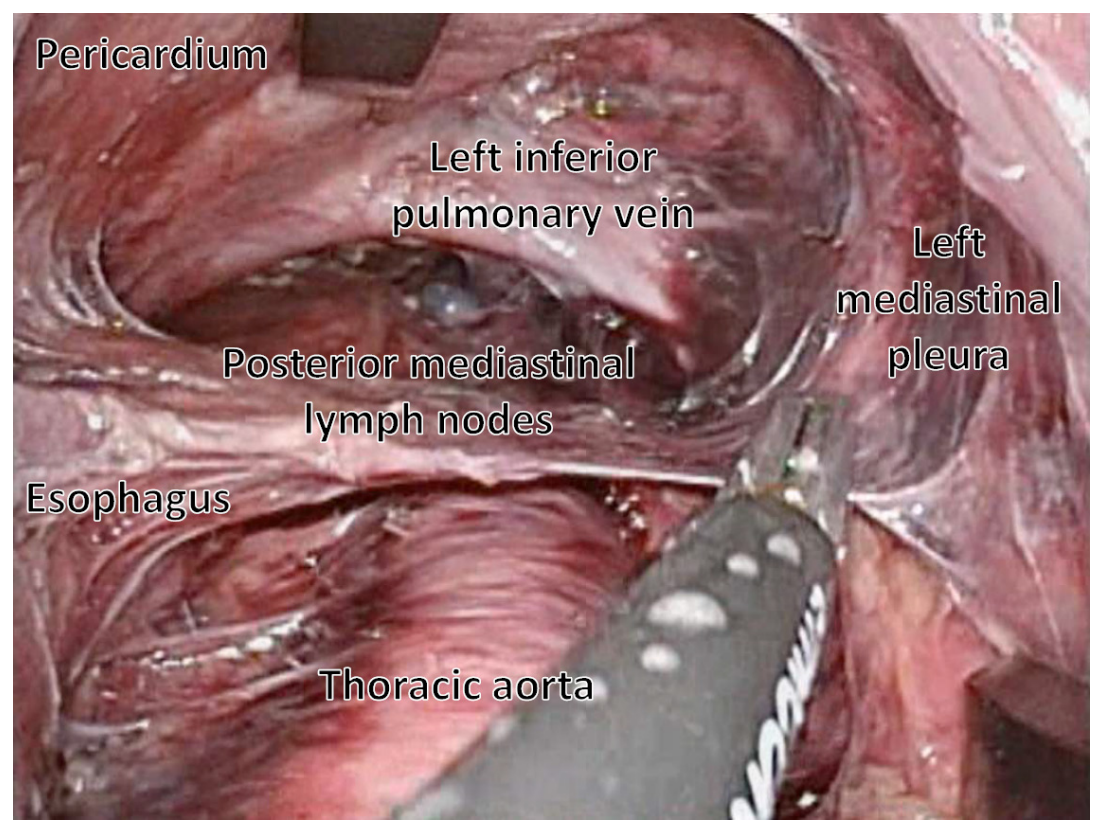

Figure 6. En bloc dissection of posterior mediastinal lymph nodes. The anterior and posterior sides of the posterior mediastinal lymph nodes were both dissected. These lymph nodes were lifted in a sheet-like form and cut along the borderline of the left mediastinal pleura

separated from the right main bronchus and tracheal bifurcation. The middle and lower mediastinal lymph nodes were dissected en bloc.

\section{Pitfall}

In this step, a detailed understanding of $3 \mathrm{D}$ images of the azygos vein is essential. At the lower mediastinal level, the azygos vein is located on the left side [Figure 7A]. However, at the middle mediastinal level, its position gradually changes to the right side [Figure $7 \mathrm{~B}$ ]. It then flows into the superior vena cava at the 


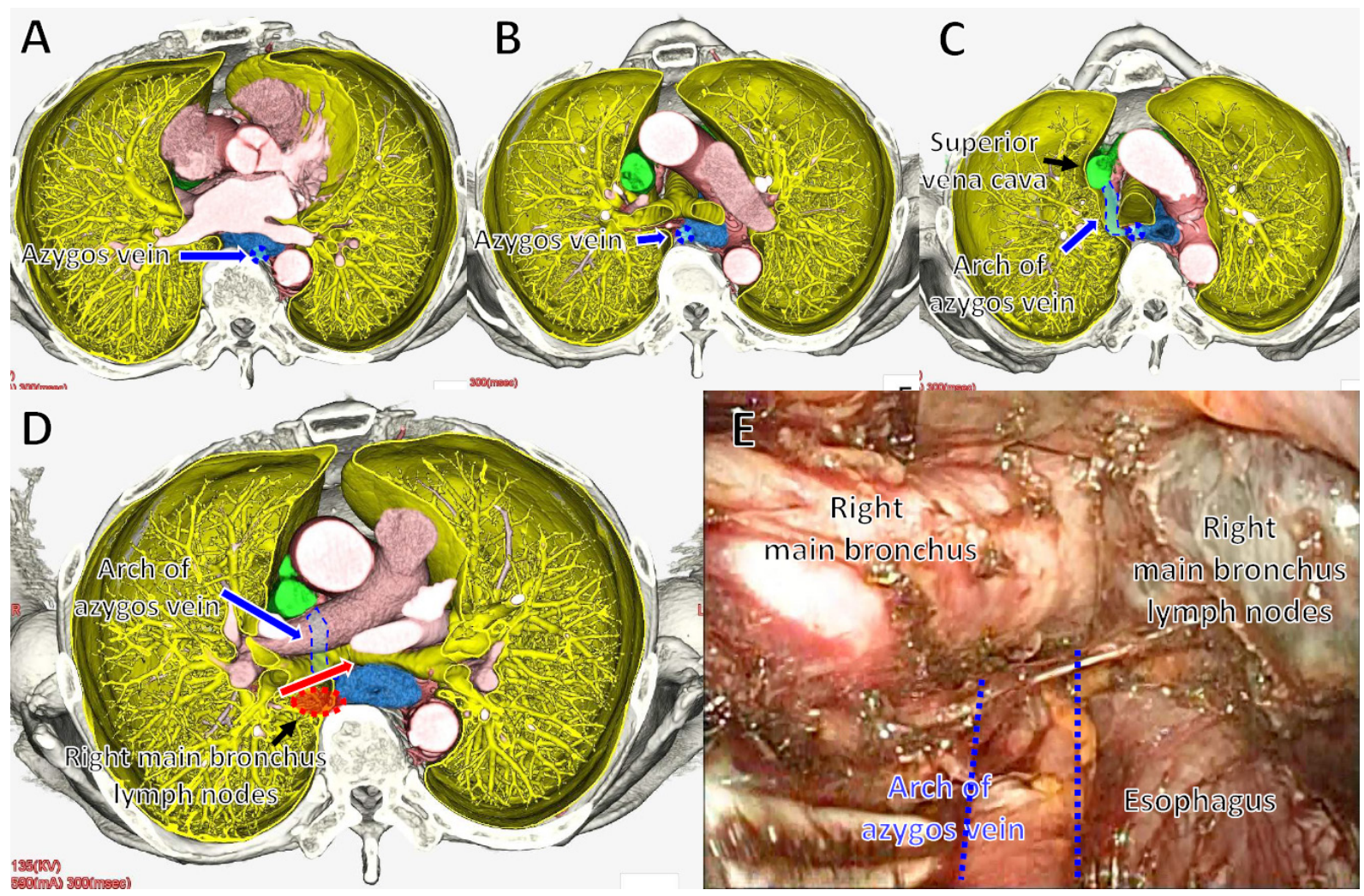

Figure 7. Pitfall around the azygos vein. At the lower mediastinal level, the azygos vein (blue arrow) is locates on the left side (A); at the middle mediastinal level, the position of the azygos vein (blue arrow) gradually changes to the right side (B); the arch of the azygos vein (blue arrow) flows into the superior vena cava at the cranial side of the right main bronchus (C); in right main bronchus lymph node (red circle) dissection, these lymph nodes are resected from the right main bronchus (red arrow). At this point, there is the risk of damage to the arch of the azygos vein (blue arrow) (D); to avoid damage, it is important to identify the arch of the azygos vein (blue dotted lines) at the dorsal side of the right main bronchus prior to right main bronchus lymph node dissection (E)

cranial side of the right main bronchus [Figure $7 \mathrm{C}$ ]. In our procedure for right main bronchus lymph node dissection, after the ventral and caudal sides were separated, these lymph nodes were resected from the right main bronchus. At this point, there was a risk of damage to the arch of the azygos vein [Figure 7D]. To avoid this, it was important to identify the arch of the azygos vein at the dorsal side of the right main bronchus prior to right main bronchus lymph node dissection [Figure $7 \mathrm{E}$ ]. We also avoided damaging the membranous portion of the right main bronchus at this point.

\section{DISCUSSION}

Recent advances in the development of surgical devices and the standardization of operative procedures have resolved the conventional limitations associated with transmediastinal esophagectomy, such as difficulties maintaining a surgical field and operability. We previously reported the significance of transmediastinal radical esophagectomy as a minimally invasive surgery ${ }^{[10-13,16]}$. This procedure initially reduced the incidence of postoperative respiratory complications because neither thoracotomy nor twolung ventilation is performed. The total operative time may have been decreased because a change in position during surgery was not necessary in this approach. We previously compared the treatment outcomes of 84 patients with esophageal cancer who underwent mediastinal lymph node dissection by the laparoscopic transhiatal approach with those of 75 patients who underwent dissection by right thoracotomy ${ }^{[11]}$. The total operative time was significantly shorter in patients treated with the laparoscopic transhiatal approach $(332.4 \pm 106.2 \mathrm{~min})$ than in those treated with right thoracotomy $(435.7 \pm 98.0 \mathrm{~min})^{[11]}$. Furthermore, a magnified view of the deep mediastinal space using a mediastinoscope decreased the total 
operative bleeding and improved the quality of lymphadenectomy. We also found that total operative bleeding was significantly less in patients treated with the laparoscopic transhiatal approach $(216.2 \pm 193.1 \mathrm{~mL})$ than in those treated with right thoracotomy $(549.5 \pm 390.4 \mathrm{~mL})$, and that the total number of resected lymph nodes did not significantly differ between the two groups (laparoscopic transhiatal approach: $35.9 \pm$ 16.0/right thoracotomy: $40.1 \pm 20.3)^{[11]}$.

On the other hand, a detailed understanding of mediastinoscopic esophagectomy is essential for the success of this procedure. The narrow mediastinal surgical space needs to be secured by appropriate retraction and pneumomediastinal pressure in this method. In addition, we sequentially expose the mediastinal organs using a long surgical device, and, thus, this surgery is similar to "tunnel construction". A detailed understanding of the $3 \mathrm{D}$ anatomy of the mediastinum is important. We routinely construct $3 \mathrm{D}$ images from CT scans and attempt to recognize the specific anatomy of major vessels preoperatively. A detailed understanding of pitfalls is indispensable to ensure safety, and the development of procedures to overcome the pitfalls of this approach, such as the tangential view, is needed.

Robot-assisted transmediastinal radical esophagectomy was recently reported to achieve a better quality of life than open esophagectomy in both retrospective and prospective studies ${ }^{[19-2]}$. Larger studies and prospective analyses are needed for comparisons between robotic and laparoscopic transhiatal approaches. In the future, the development of novel instruments, such as small-caliber devices with multiple joints, and lightweight robotic single-port techniques may be key innovations in transmediastinal radical esophagectomy.

\section{CONCLUSION}

Laparoscopic transhiatal esophagectomy provided a good surgical view and safe en bloc mediastinal lymph node dissection in patients with esophageal cancer. The standardization of surgical procedures and a detailed understanding of the mediastinal 3D anatomy and specific pitfalls are important for the success of this approach.

\section{DECLARATIONS}

\section{Authors' contributions}

Wrote the manuscript: Shiozaki A, Fujiwara H, Otsuji E

Performed surgeries: Shiozaki A, Fujiwara H, Konishi H

Designed the research: Shiozaki A, Fujiwara H, Konishi H, Shimizu H, Kudou M, Arita T, Kosuga T, Morimura R, Kuriu Y, Ikoma H, Kubota T, Okamoto K, Otsuji E

\section{Availability of data and materials}

Not applicable.

\section{Financial support and sponsorship}

None.

\section{Conflicts of interest}

All authors declared that there are no conflicts of interest.

\section{Ethical approval and consent to participate}

Our work conforms to the guidelines set forth in the Helsinki Declaration concerning human and animal rights, and we followed the policy concerning informed consent. The study of this surgical procedure was reviewed and approved by the Kyoto Prefectural University of Medicine Institutional Review Board. 


\section{Consent for publication}

Written informed consent for publication was obtained.

\section{Copyright}

(c) The Author(s) 2020.

\section{REFERENCES}

1. Tachimori Y, Ozawa S, Numasaki H, Matsubara H, Shinoda M, et al; Registration Committee for Esophageal Cancer of the Japan Esophageal Society. Efficacy of lymph node dissection by node zones according to tumor location for esophageal squamous cell carcinoma. Esophagus 2016;13:1-7.

2. Udagawa H, Ueno M, Shinohara H, Haruta S, Kaida S, et al. The importance of grouping of lymph node stations and rationale of threefield lymphoadenectomy for thoracic esophageal cancer. J Surg Oncol 2012;106:742-7.

3. Tachimori Y, Ozawa S, Numasaki H, Ishihara R, Matsubara H,et al.; Registration Committee for Esophageal Cancer of the Japan Esophageal Society. Comprehensive registry of esophageal cancer in Japan, 2012. Esophagus 2019;16:221-245.

4. Orringer MB, Sloan H. Esophagectomy without thoracotomy. J Thorac Cardiovasc Surg 1978;76:643-53.

5. DePaula AL, Hashiba K, Ferreira EA, de Paula RA, Grecco E. Laparoscopic transhiatal esophagectomy with esophagogastroplasty. Surg Laparosc Endosc 1995;5:1-5.

6. Bonavina L, Bona D, Binyom PR, Peracchia A. A laparoscopy-assisted surgical approach to esophageal carcinoma. J Surg Res 2004;117:52-7.

7. Montenovo MI, Chambers K, Pellegrini CA, Oelschlager BK. Outcomes of laparoscopic-assisted transhiatal esophagectomy for adenocarcinoma of the esophagus and esophago-gastric junction. Dis Esophagus 2011;24:430-6.

8. Shiozaki A, Fujiwara H, Murayama Y, Komatsu S, Kuriu Y, et al. Posterior mediastinal lymph node dissection using the pneumomediastinum method for esophageal cancer. Esophagus 2012;9:58-64.

9. Shiozaki A, Fujiwara H, Murayama Y, Komatsu S, Kuriu Y, et al. Hand-assisted laparoscopic transhiatal approach for mediastinal esophageal duplication cyst resection. Esophagus 2012;9:247-51.

10. Shiozaki A, Fujiwara H, Murayama Y, Komatsu S, Kuriu Y, et al. Perioperative outcomes of esophagectomy preceded by the laparoscopic transhiatal approach for esophageal cancer. Dis Esophagus 2014;27:470-8.

11. Shiozaki A. Fujiwara H, Konishi H, Arita T, Kosuga T, et al. Hand-assisted technique beneficial for laparoscopic transhiatal esophagectomy with en-bloc dissection of middle and lower mediastinal lymph nodes: roles of the operator's left hand. Esophagus 2017; 14:138-45

12. Shiozaki A, Fujiwara H, Konishi H, Morimura R, Murayama Y,et al. Novel technique for dissection of subcarinal and main bronchial lymph nodes using a laparoscopic transhiatal approach for esophageal cancer. Anticancer Res 2013;33:2577-85.

13. Fujiwara H, Shiozaki A, Konishi H, Komatsu S, Kubota T,et al. Hand-assisted laparoscopic transhiatal esophagectomy with a systematic procedure for en bloc infracarinal lymph node dissection. Dis Esophagus 2016;29:131-8.

14. Fujiwara H, Shiozaki A, Konishi H, Kosuga T, Komatsu S, et al. Single-Port mediastinoscopic lymphadenectomy along the left recurrent laryngeal nerve. Ann Thorac Surg 2015;100:1115-7.

15. Fujiwara H, Shiozaki A, Konishi H, Otsuji E. Mediastinoscope and laparoscope-assisted esophagectomy. J Vis Surg 2016;2:125.

16. Fujiwara H, Shiozaki A, Konishi H, Kosuga T, Komatsu S, et al. Perioperative outcomes of single-port mediastinoscope-assisted transhiatal esophagectomy for thoracic esophageal cancer. Dis Esophagus 2017;30:1-8.

17. Fujiwara H, Shiozaki A, Konishi H, Otsuji E. Transmediastinal approach for esophageal cancer: A new trend toward radical surgery. Asian J Endosc Sur 2019;12:30-6.

18. Shiozaki A, Fujiwara H, Konishi H, Kosuga T, Komatsu S, et al. Successful subcarinal dissection using a laparoscopic transhiatal approach for esophageal cancer with an anomalous pulmonary vein. Gen Thorac Cardiovasc Surg 2016;64:239-42.

19. Yoshimura S, Mori K, Yamagata Y, Aikou S, Yagi K, et al. Quality of life after robot-assisted transmediastinal radical surgery for esophageal cancer. Surg Endosc 2018;32:2249-54.

20. Sugawara K, Yoshimura S, Yagi K, Nishida M, Aikou S, et al. Long-term health-related quality of life following robot-assisted radical transmediastinal esophagectomy. Surg Endosc 2020;34:1602-11.

21. Seto Y. Essential Updates 2018/2019: Essential Updates for esophageal cancer surgery. Ann Gastroenterol Surg 2020;4:190-4.

22. Seto Y, Mori K, Aikou S. Robotic surgery for esophageal cancer: merits and demerits. Ann Gastroenterol Surg 2017;1:193-8. 